\title{
La innovación y la importancia de la cercanía o la lejanía: el caso de Microsoft Consulting Services
}

\author{
Fernando Moliní \\ Departamento de Geografía, Universidad Autónoma de Madrid \\ fernando.molini@uam.es \\ Eduardo Estrada \\ Facultad de Psicología, Universidad Autónoma de Madrid \\ eduardo.estrada@uam.es
}

La innovación y la importancia de la cercanía o la lejanía: el caso de Microsoft Consulting Services (Resumen)

Se hacen diversos análisis teóricos a partir de la revisión crítica de la bibliografía más relevante, particularmente sobre la situación de Microsoft respecto a otras empresas tecnológicas y sobre la relación entre territorio e innovación. El núcleo principal del artículo lo constituye la realización de una encuesta a los consultores relacionados con la informática de Microsoft Consulting Services (MCS). Respecto a los ámbitos geográficos a los que pertenecen otras empresas con las que tienen relaciones que les ayudan a ser más innovadores, las respuestas más seleccionadas fueron la Unión Europea, España y otras zonas desarrollas del mundo no hispanoparlantes, por este orden. Para la innovación parece fundamental combinar las relaciones externas lejanas y potencialmente dispersas, en que es posible acceder a lo mejor, con las locales que son próximas, concentradas y accesibles. Los empleados de MCS parece que combinan ambas vertientes de la innovación, la global y la local.

Palabras clave: innovación, territorio, empresas tecnológicas, Microsoft.

Innovation and the relevance of closeness or remoteness: the case of Microsoft Consulting Services (Abstract)

Several theoretical analyses are carried out, starting with the critical review of the most relevant literature, particularly over the situation of Microsoft with respect to other technology firms and on the relationship between territory and innovation. The core of the paper is a survey of the programmers and computerrelated consultants at Microsoft Consulting Services (MCS). Regarding the geographical areas of other companies with which they have relationships that help them to be more innovative, the most frequently selected responses were to the European Union, Spain and other developed areas of the non Spanishspeaking world, in that order. For innovation it is essential to combine external, distant relationships that are potentially dispersed, and that give access to the best professionals, with concentrated and accessible local relationships. Employees at MCS seem to combine both aspects of innovation, global and local. 
Key words: innovation, territory, technology business, Microsoft.

Con esta investigación pretendemos un doble objetivo, por una parte que, a ser posible, sirva para mejorar la innovación de la empresa a la que se aplica y, por otra, avanzar en el conocimiento de la relación entre distancia e innovación a partir del análisis de su caso. Que sepamos, se aborda por primera vez el investigar los ámbitos geográficos de la innovación de una empresa con empleados dedicados a la programación y consultoría relacionadas con la informática. La relación entre innovación y distancia es particularmente interesante de estudiar en este tipo de profesionales, porque pueden realizar con menos dificultad su actividad a distancia. Esto se debe a que dominan las tecnologías de la telecomunicación y a que su trabajo se puede transportar por ellas fácilmente. En su caso, el territorio ejerce una fricción menor que en otro tipo de trabajos. Por lo tanto, si la cercanía les fuese importante para la innovación, lo sería no tanto porque no tengan otra alternativa, sino por sus ventajas intrínsecas.

Dentro de las empresas del sector, se quiso buscar al menos un caso de estudio que fuese particularmente relevante. Se optó por el departamento de Microsoft Consulting Services de la empresa Microsoft Ibérica, por su carácter emblemático y porque fue la empresa relacionada con la programación y la consultoría informática que tuvo una mejor predisposición para colaborar con la universidad en la investigación. Para otras empresas el proceso de elaborar la encuesta era de menor interés o más complicado. Por ejemplo, esto último fue el caso de la filial de otra multinacional, que primero tenía que traducir la propuesta al idioma de la casa matriz y después lograr que ésta aprobase su realización, un proceso que le resultaba demasiado complicado como para intentarlo.

\section{Microsoft: una empresa altamente innovadora, pero recientemente superada}

El caso de estudio es particularmente relevante y adecuado. En la bibliografía científica hay numerosos artículos basados en investigaciones sobre Microsoft ${ }^{1}$, así como son muy abundantes los libros referidos como único caso de estudio a Microsoft ${ }^{2}$. Los artículos científicos mencionados sobre Microsoft tratan temáticas muy diversas, mientras que los libros se centran más en la innovación.

Resulta muy singular el que los empleados de una empresa dedicada a la programación y consultoría informática hayan respondido a una encuesta sobre la innovación. Existe el precedente de libros cuyos autores realizan entrevistas a directivos de Microsoft sobre la innovación $^{3}$, pero no hemos encontrado trabajos en que se haga una encuesta a sus empleados más cualificados, particularmente con la finalidad de investigar la relación de la innovación con la cercanía y la distancia.

\footnotetext{
${ }^{1}$ Behanji, 2012; Heath y Bell, 2006; van Heck et al., 2012; Klein y otros, 2005; Moyle, 2003; Sandler y P. Blank,

${ }^{2}$ Barr, 2000; Bank, 2001; Foley, 2008; Heilemann, 2003; Manes y Andrews, 1994; Slater, 2004; Stross, 1997; Toulouse, 2010 Wallace y Erickson, 1993.

${ }^{3}$ Cusumano, 2000; Cusumano, 2012; Manes y Andrews, 1994; Slater, 2004 y Stross, 1997.
} 
El caso de estudio es del máximo interés por muchas razones, particularmente por la importancia de la empresa y los problemas a los que se enfrenta. Para Andrew y Sirkin ${ }^{4}$ "Microsoft es la empresa más innovadora de todos los tiempos". Dan mucha importancia a que "de 1985 a 2004, los ingresos aumentaron en una tasa de crecimiento anual compuesto del 34 por ciento, mientras que las ganancias aumentaron anualmente en un 36 por ciento". Microsoft es una de las mayores empresas globales y uno de las más admiradas, según evaluaciones realizadas por criterios múltiples, que van desde la innovación a la responsabilidad social corporativa. Pero también es una de las compañías que recientemente ha perdido algunas de las grandes batallas tecnológicas. Arthur $^{5}$ resalta que Microsoft está perdiendo la guerra digital, fundamentalmente frente a Apple y Google, si bien habría que decir que por el momento, puesto que ha habido grandes casos de recuperación de empresas de TIC. Algunos de sus trabajadores hacen un buen balance de su paso por la empresa, por ejemplo Toulouse ${ }^{6}$. Pero muchos otros pensaron que ya no era el mejor lugar para trabajar, como lo indica el que más de 100 empleados de Microsoft se marcharon a trabajar a Google ${ }^{7}$.

El problema es que Microsoft ha sido posiblemente la mejor empresa entre las mejores empresas y ya no es así, aunque todavía se mantiene en muy buena posición. Como dice Cusumano ${ }^{8}$, las empresas que permanentemente lo hacen bien necesitan reinventarse continuamente. Esto es muy difícil cuando se ha estado en una posición absolutamente dominante, como le ha sucedido a Microsoft. El autor advierte que sean cuales sean los secretos del éxito o de las buenas prácticas que las empresas dominen en un momento dado, esas ventajas es probable que se conviertan en obsoletas o menos efectivas a medida que las condiciones y la tecnología evolucionan, y a medida en que los competidores mejoran. Pero, incluso desde antes de que se haya visto superada por Apple y Google, Microsoft no era una empresa arrogante que pensase que eran los mejores. Manes y Andrews ${ }^{9}$ recogen el testimonio de algunos directivos a este respecto, particularmente el de Jeff Harbers, que dice que siempre creen que lo pueden hacer mejor, que tienen una baja confianza en que son los mejores y que siempre creen que hay alguien en el horizonte haciéndolo diez veces mejor. El fundador de Microsoft también lo afirmó: "El éxito es un malísimo profesor. Seduce a la gente inteligente y la lleva a pensar que no puede perder. También es una guía poco fiable para el futuro. Lo que parece ser el plan de negocios perfecto o la última tecnología hoy, puede estar obsoleto dentro de poco". Y recomienda "la necesidad de iniciar tendencias en lugar de seguirlas"10. Un problema añadido para generar nuevas oportunidades y para adaptarse a las nuevas circunstancias es que los cambios son cada vez más rápidos. Microsoft lanzó en 1981 su exitoso sistema operativo MS-DOS. Hasta 1990 tan solo realizó mejoras evolutivas. En ese año introdujo Windows $3.0^{11}$. Este ritmo lento de innovación ya no se lo puede permitir ninguna empresa tecnológica.

\footnotetext{
${ }^{4}$ Andrew y Sirkin, 2008.

${ }^{5}$ Arthur, 2012.

6 Toulouse, 2010.

7 Arthur, 2012.

${ }^{8}$ Cusumano, 2012.

${ }^{9}$ Manes y Andrews, 1994.

${ }^{10}$ Gates, 1995.

${ }^{11}$ Cusumano, 2000.
} 
Ser permanentemente proactivo es muy difícil. Stross ${ }^{12}$ señala que ninguna compañía informática ha sido capaz de ser la predominante en dos eras tecnológicas distintas, por mucho que lo hayan intentado. IBM se vio sobrepasada por Digital Equipment Corporation (DEC) en la era de las microcomputadoras; DEC por Microsoft en la era de los ordenadores personales; y Microsoft por Google en la era de Internet. Pero las empresas de nuevas tecnologías pueden resurgir, como le ha sucedido a IBM o, sobre todo, a Apple. El volumen de negocios de esta última había pasado de 11.000 millones de dólares en 1995 a 7.000 en 1997. En el primer trimestre de 1997 tuvo pérdidas de 708 millones de dólares. En aquella época logró un acuerdo de colaboración con la empresa dominante de momento, Microsoft, que adquirió acciones de Apple por 150 millones de dólares y desarrolló versiones de Office para los Mac, a cambio de que su Internet Explorer se convirtiese en el navegador por defecto de los ordenadores de Apple. Parecía imposible que Apple pudiese algún día superar a Microsoft. Sin embargo, en el año 2010 la capitalización bursátil de Apple sobrepasó a la de Microsoft ${ }^{13}$. Y en el 2012 lo haría la de Google $^{14}$.

Microsoft empezó a fallar no tanto por hacerlo mal sino, sobre todo, por no ser capaz de generar muchas de las nuevas grandes oportunidades de negocio que eran posibles en su campo. El que Microsoft esté perdiendo posiciones respecto a la competencia le obliga a innovar en mayor medida, en un grado tal que sea capaz de superarla. Esto hace que la presente investigación sobre la innovación en una de las áreas de consultoría de la filial española de Microsoft sea particularmente oportuna.

\section{Microsoft Ibérica: un excelente caso de estudio}

Microsoft Ibérica es un relevante tema de investigación, que va más allá de ser una filial de una multinacional estadounidense, porque tiene importancia y singularidad en sí misma. Es una compañía puntera en España en nuevas tecnologías. La empresa tenía 639 empleados en el año $2011^{15}$, aunque este número puede que no sea del todo preciso. Otras fuentes sitúan sus trabajadores algo por encima. Así, Actualidad Económica ${ }^{16}$ los estima en 680 para ese mismo año. Los empleados han ido aumentando de manera continuada y rápida, hasta algo después del inicio de la crisis económica, en que han sufrido un ligero retroceso. Así, tenía 325 trabajadores en 2003, 664 en 2009 y 639 en $2011^{17}$. Se ha estimado que Microsoft Ibérica genera 40.000 empleos indirectos y que, por cada euro que ingresa en España, las empresas relacionadas con ella generan $11,10 €^{18}$. Su facturación ha seguido una evolución parecida a la del empleo. En 2003 fue de 91,8 millones de \$, en 2008 tuvo un máximo de 230,8 millones de \$, en 2010 sufrió un descenso hasta los 190,8 millones de \$ y en 2011 experimentó una cierta recuperación, con 227,8 millones de $\$^{19}$. Aunque es una empresa plenamente solvente, se resiente de la crisis

\footnotetext{
12 Stross, 2009.

${ }^{13}$ Ichbiah, 2011.

${ }^{14}$ Europa Press, 2012.

${ }^{15}$ BVD/ID\&B, 2013.

${ }^{16}$ Actualidad Económica, 2012.

${ }^{17}$ BVD/ID\&B, 2013.

${ }^{18}$ Sánchez, H., 2009.

19 BVD/ID\&B, 2013.
} 
económica y ello hace que sea un momento particularmente adecuado para investigar cómo intentar que sea más innovadora.

Otra de las razones por la que merece la pena investigar a Microsoft Ibérica es que se trata de una de las mejores empresas para trabajar en España, situándose con frecuencia en primera posición. En la categoría de entre los 500 y 1.000 empleados, en 2013 se sitúa en cuarta posición, en 2012 en la primera, en 2011 también en la primera, en 2010 en la segunda, etc ${ }^{20}$. El prestigio de Microsoft es tan elevado que su aval sirve para considerar que un proyecto tiene valor internacional. Es el caso del Centro de Educación Infantil y Primaria del núcleo rural de Ariño, que se convirtió en uno de los centros educativos pioneros a nivel mundial por su incorporación y utilización de las TIC. Esto contribuyó a frenar su despoblamiento y a su desarrollo socioeducativo. Se considera que es un ejemplo internacional porque Microsoft ha referenciado el proyecto como modelo a seguir ${ }^{21}$.

\section{Metodología}

El análisis de de la innovación en Microsoft Ibérica ha consistido en enviar una encuesta por correo electrónico a sus empleados que sean programadores o consultores relacionados con la informática, entendidos en un sentido amplio. La encuesta se centró en el departamento de Microsoft Consulting Services (MCS) por cumplir con estas características. Su objetivo principal es ayudar a los clientes más importantes a implementar soluciones con su tecnología. No se dedican pues a investigación y desarrollo. El grado de innovación que pueden introducir está relacionado con sus procesos internos y con las propuestas de las mejores soluciones posibles de cara a atender a sus clientes. Los requisitos planteados los cumplían 75 empleados, que estaban entre los más cualificados de la empresa. El Departamento de Recursos Humanos de Microsoft Ibérica les envió un correo electrónico con el enlace a la encuesta el 12 de abril de 2013. En una primera fase contestaron 24 de ellos. Se les mandó un segundo correo electrónico, a finales de abril de 2013, recordándoles el interés de la investigación. En esta ocasión contestaron 12 empleados más, en total 36, por lo que la tasa de respuesta ha sido del 48 por ciento. La encuesta constaba de 21 preguntas cerradas y de una abierta. Se realizó a través de SurveyMonkey.

Para fomentar el que Microsoft Ibérica colaborase en la investigación y mandase el cuestionario a sus empleados, se le ofreció la realización de una encuesta gratuita que tuviese una doble finalidad: por una parte, que potencialmente sirviese para mejorar su innovación y, por otra, que permitiese averiguar algunos de los elementos de la relación entre territorio e innovación. La encuesta a los empleados se completó con información proporcionada por la dirección de la empresa, en algunos casos puntuales que se mencionan.

Las hipótesis principales de la investigación son:

1. Un departamento puntero como MCS tiene pocos aspectos importantes para mejorar respecto a la innovación.

2. Las relaciones externas son importantes para la innovación.

\footnotetext{
${ }^{20}$ GPTWE, 2013.

${ }^{21}$ Elboj et. al., 2013.
} 
Los profesionales que pueden trabajar más desapegados del territorio, como los empleados de MCS, aprovechan esta oportunidad y lo hacen a distancias largas.

A Las relaciones con otras empresas son frecuentes y se producen sobre todo fuera de España. B Las relaciones con universidades son frecuentes y se producen sobre todo fuera de España.

3. El disponer cerca de una alta concentración de empresas de alta tecnología es beneficioso para la innovación.

4. Se preferirá un socio ligeramente mejor, ubicado más lejos, que uno ligeramente peor, pero ubicado más cerca.

5. Los empleados que innovan más colaboran con empresas ubicadas a distancias mayores.

6. Los empleados que innovan más colaboran con universidades ubicadas a distancias mayores.

7. Los empleados que dominan más el inglés (nivel alto o muy alto) trabajan más con empresas de territorios no hispanoparlantes que los que tienen un nivel medio o inferior.

8. Los empleados que dominan más el inglés (nivel alto o muy alto) son más innovadores que los que tienen un nivel medio o inferior.

9. Los empleados que teletrabajan innovan menos que los que no teletrabajan.

La metodología de estudio de casos se ha utilizado muy ampliamente y está muy contrastada. No obstante, tiene el inconveniente de que no permite hacer generalizaciones. A cambio, se estudia una situación con más profundidad y, a partir de ello, puede permitir generar un gran número de ideas. Por otro lado, lo que sí se puede generalizar en mayor medida es la metodología empleada, que puede utilizarse en muchos otros estudios de casos y de otro tipo, y que tal vez sea novedosa. Particularmente algunas cuestiones planteadas, como evaluar si ante un nuevo reto primero se piensa por sí mismo y luego se acude a otras fuentes, o saber si se prefiere a un socio ligeramente mejor, ubicado más lejos, que a uno peor pero más cercano. Asimismo, la peculiaridad de haber unido dos tipos de preguntas: en primer lugar, aquellas cuyo objetivo es mejorar la innovación de la empresa y, en segundo lugar, aquellas cuyo objetivo es conocer el tema que más interesa al investigador y que puede tener menos utilidad práctica inmediata, que en este caso es la relación entre la innovación y la distancia.

La encuesta es un método de investigación muy utilizado, pero no deja de recibir críticas. Méndez y et al. ${ }^{22}$ ven problemas a las investigaciones realizadas mediante encuestas y entrevistas en ámbitos locales concretos, que a veces se pueden generalizar a otras situaciones. Especifican dos problemas: que son poco exigentes en la identificación de lo que es verdadera innovación y que utilizan una gran variedad de metodologías, lo que las hace poco comparables. Pero también resaltan que son de gran interés para la comprensión de los procesos.

Un punto débil de utilizar encuestas es que se basa en la opinión de los que responden y no se puede saber en qué grado son sinceros al emitirla. Al ser anónimas, se evita en gran medida el que haya razones para mentir, pero nunca se eliminan del todo. En esta encuesta ha habido respuestas muy sinceras, como la del empleado que dice que no ha comunicado una innovación a la empresa para utilizarla en beneficio personal. El problema es que el encuestado tienda a contestar en un grado excesivamente alto aquello que le proporciona una mejor imagen, y en un

\footnotetext{
${ }^{22}$ Méndez y et al., 2008.
} 
grado excesivamente bajo en caso contrario. Lee y Woodliffe ${ }^{23}$ dicen que hay un sesgo de deseabilidad social que puede distorsionar las respuestas cuando éstas dan unos valores más altos a lo que es socialmente deseable y viceversa. Sin embargo, también afirman que las encuestas por correo postal o electrónico, como la de esta investigación, pueden reducir esta tendencia. Aquí el sesgo de deseabilidad social se daría sobre todo en la pregunta "Indique el número de innovaciones (mejoras relevantes de productos, procesos u otras que sean o tengan potencial de ser un éxito comercial) que usted haya generado en los últimos 3 años y que considere que son pioneras a escala internacional". El sesgo de deseabilidad social no parece que haya afectado a las respuestas, puesto que la mayoría quedan en las dos categorías más bajas: el 31,43 por ciento contesta que ninguna y el 45,71 por ciento que de 1 a 2 . Por el contrario, solo el 5,71 por ciento contesta que de 5 a 6 y un porcentaje idéntico que 6 o más. Puede que en las respuestas más altas haya una cierta exageración, pero en su conjunto parecen razonables. En la interpretación de todos los datos debe de tenerse en cuenta que la encuesta no está hecha a un departamento de investigación y desarrollo, sino a uno de consultores. Esta advertencia no se vuelve a repetir a lo largo de artículo, pero es aplicable en todo él. En este sentido no pueden considerarse bajas las respuestas obtenidas en cuanto al número de innovaciones.

\section{Mejorar la innovación de MCS}

A continuación se comentan las preguntas y respuestas que pretendían evaluar la innovación en MCS. La hipótesis 1 sostiene que "un departamento puntero como MCS tiene pocos aspectos importantes para mejorar respecto a la innovación". Las respuestas a varias preguntas tienden a confirmarlo.

\section{Cuadro 1 \\ Grado en que la empresa motiva a sus empleados a ser innovadores}

\begin{tabular}{|lcc|} 
Respuestas & $\%$ & Número \\
\hline Muy alto & 22,2 & 8 \\
\hline Alto & 55,6 & 20 \\
\hline Medio & 19,4 & 7 \\
\hline Bajo & 2,8 & 1 \\
Muy bajo. & 0,0 & 0 \\
\hline Responden & & 36 \\
\hline No responden & & 0 \\
\hline Fuente: elaboración propia & &
\end{tabular}

El cuadro 1 recoge las respuestas a la pregunta "¿en qué grado considera que la empresa motiva a sus empleados a ser innovadores?". Las repuestas reflejan una alta motivación para ser innovadores.

${ }^{23}$ Lee y Woodliffe, 2010. 
A la pregunta "¿ha recibido formación específica sobre la innovación o ha participado en sesiones de tormentas de ideas?", hubo 36 respuestas. La mayoría contesta que sí (el 61,11 por ciento), pero hay un porcentaje relativamente importante que no (el 38,89 por ciento).

El cuadro 2 refleja las respuestas a la pregunta "¿en qué grado considera que el canal que existe en su empresa para transmitir ideas innovadoras es adecuado?". El resultado es bueno, pero hay un cierto margen para mejorar.

\section{Cuadro 2}

\begin{tabular}{|c|c|c|}
\hline \multicolumn{3}{|c|}{$\begin{array}{l}\text { En qué grado es adecuado el canal que existe en } \\
\text { la empresa para transmitir ideas innovadoras }\end{array}$} \\
\hline Respuestas & $\%$ & Número \\
\hline Muy adecuado & 16,7 & 6 \\
\hline Adecuado & 47,2 & 17 \\
\hline Medio & 22,2 & 8 \\
\hline Inadecuado & 11,1 & 4 \\
\hline Muy inadecuado & 2,8 & 1 \\
\hline Responden & & 36 \\
\hline No responden & & 0 \\
\hline
\end{tabular}

A la pregunta "¿la empresa penaliza de alguna manera a los empleados o departamentos que tienen ideas nuevas y fracasan, por ejemplo rechazando en mayor medida posteriores propuestas de la misma persona o departamento?" Hubo 36 respuestas y la práctica totalidad (el 94,44 por ciento) contestó que no.

A la pregunta " $¿$ alguna vez ha tenido una idea sobre su trabajo que consideraba innovadora y no la ha comunicado a su empresa?", hubo 36 respuestas. El 75 por ciento contestó que no y el 25 por ciento que sí. Se señaló que, si se había contestado sí a la anterior pregunta, se indicasen los motivos por los que no se comunicó su innovación, pudiendo señalar todos los que procedan.

Hubo 8 respuestas, con las siguientes opciones, expuestas de mayor a menor número de respuestas: "no hay un canal adecuado para comunicarla" (el 62,50 por ciento de los que responden); "comunicarla me habría supuesto más trabajo y complicarme la vida" (el 50 por ciento); "alguna vez había comunicado una idea innovadora y no me la tomaron suficientemente en serio" (el 25 por ciento); "por timidez o falta de confianza en mí mismo" (el 25 por ciento).

La opción "no creía que fuese a ser bien recibida" no obtuvo ninguna respuesta. En "otras" se señalaron dos causas: no suscitaría interés si no hay beneficio a corto plazo y he preferido desarrollar estas ideas para beneficio personal.

El cuadro 3 refleja las respuestas a la pregunta "¿en qué grado habla, escribe y entiende inglés lo suficientemente bien como para relacionarse con pocas dificultades con angloparlantes?". El nivel de dominio del inglés es muy elevado. 


\begin{tabular}{|c|c|c|}
\hline \multicolumn{3}{|c|}{$\begin{array}{c}\text { Cuadro } 3 \\
\text { Nivel de conocimiento de inglés }\end{array}$} \\
\hline Respuestas & $\%$ & Número \\
\hline Muy alto & 22,2 & 8 \\
\hline Alto & 55,6 & 20 \\
\hline Medio & 22,2 & 8 \\
\hline Bajo & 0,0 & 0 \\
\hline Muy bajo & 0,0 & 0 \\
\hline Responden & & 36 \\
\hline No responden & & 0 \\
\hline
\end{tabular}

Fuente: elaboración propia

Relacionamos estas respuestas con las de la pregunta "indique el número de innovaciones (mejoras relevantes de productos, procesos u otras que sean o tengan potencial de ser un éxito comercial) que usted haya generado en los últimos 3 años y que considere que son pioneras a escala internacional". Así analizamos si los empleados que dominan más el inglés (nivel alto o muy alto) son más innovadores que los que tienen un nivel medio o inferior (hipótesis 9). Aplicando la prueba Chi-Cuadrado sobre independencia de proporciones, se obtuvo un valor de $\chi^{2}=9,071(g l=8, p=, 336)$. Este resultado no alcanza significación estadística.

A la pregunta "¿hace esfuerzos por generar un flujo continuo de ideas innovadoras dedicando tiempo a pensar en ello todas las semanas?", hubo 36 respuestas. El 63,89 por ciento contesta que no y el 36,11 por ciento que sí. Entre las estudiadas esta es la práctica en la que MCS tal vez podría mejorar en mayor grado, si es que es aplicable a sus circunstancias. Máxime cuando de los 14 empleados que responden a la pregunta abierta "escriba cualquier mejora que propondría para que su empresa fuese más innovadora", tres responden que dedicar más tiempo para la innovación, mientras que ninguna otra respuesta se repite. La falta de tranquilidad para innovar contrasta con la de algunas empresas de la competencia, si bien hay que tener en cuenta que los encuestados en MCS son consultores, mientras que aquí se van a comparar con programadores con mayor responsabilidad en la innovación. Respecto a proporcionar tiempo libre a sus empleados para innovar, destaca la política de Google, cuyos ingenieros tienen el 20 por ciento de su tiempo para pensar e innovar libremente ${ }^{24}$. Esta práctica se denomina tiempo libre para la innovación. Su único requisito es que tienen que justificar a sus superiores lo hecho en esas horas de reflexión. Se calcula que del tiempo libre para innovar se derivan, de manera más o menos directa, más del 50 por ciento de los nuevos productos de Google ${ }^{25}$.

Respecto a la hipótesis 1 un resultado ambivalente lo proporciona las respuestas a la pregunta "ante un nuevo problema o reto que le plantean, lo primero que hace es". Hubo 36 respuestas. El 50 por ciento responde que pienso por mí mismo y escribo mis ideas antes de acudir a otras fuentes y el otro 50 por ciento que miro lo ya elaborado sobre el tema y luego escribo mis ideas. En nuestra opinión la opción que tiene más posibilidades de creatividad es la primera, porque en el segundo caso las lecturas de los demás condicionan el propio pensamiento y se hace más difícil proponer algo diferente. Si primero se piensa por uno mismo, lo más probable es que al cotejarlo con otras fuentes se descubra que casi todo lo reflexionado ya estaba previamente dicho

\footnotetext{
${ }^{24}$ Arthur, 2012.

25 Johnson, 2011.
} 
o hecho. Pero de ese pensamiento propio pueden quedar partes que no se encuentren en lo previamente existente. Esas partes podrían ser innovadoras, aunque también hay altas probabilidades de que no se han buscado bien los posibles precedentes. En cualquier caso, sirve para ejercitar en un mayor grado la mente. Hay autores que parecen no estar de acuerdo con esta idea. Foster ${ }^{26}$ propone 101 métodos para generar ideas y ninguno es el de primero pensar por uno mismo para después contrastar el pensamiento propio con otras fuentes, que a nosotros nos parece fundamental. Es más, en alguno de ellos más bien sugiere lo contrario. Asimismo, Trompenaards y Hampden-Turner ${ }^{27}$ afirman que "aquellos que desean pensar y actuar por sí mismos necesitan información con la que pensar y deben tener la sensatez de escuchar antes, so pena de llegar a una conclusión equivocada". Efectivamente, si uno piensa antes de escuchar, es muy probable que llegue a conclusiones equivocadas. Pero ello es bueno si se es consciente de que esto es muy posible de que pase, porque al contrastar las ideas propias con las fuentes y ver que no son válidas, se rectifica. La ventaja es que en el proceso habrá tenido más oportunidades de ser original y ello es un entrenamiento de gran valor.

\section{Territorio e innovación}

Numerosos autores opinan que la proximidad de las relaciones y de las redes son importantes para que las empresas sean más innovadoras, aunque con muchos matices ${ }^{28}$. La relación entre innovación y proximidad difiere según muchos aspectos, como el tamaño de las empresas. Drejer y Vinding ${ }^{29}$ dicen que las pequeñas empresas son más dependientes para la innovación del entorno local, lo que también es corroborado por Tödtling y Kaufman ${ }^{30}$. Igualmente difiere según el tipo de agentes implicados. Así, la proximidad importa particularmente en la colaboración con los intermediarios relacionados con la innovación, como centros de investigación, agencias gubernamentales o incubadoras de empresas ${ }^{31}$. En una encuesta realizada a pequeñas y medianas empresas (PYME) guipuzcoanas, Lavía et al ${ }^{32}$ encuentran que un 90,5 por ciento de las que cooperan con agentes de investigación y desarrollo lo hacen con los ubicados en la propia provincia o región, mientras que únicamente el 50 por ciento de las que cooperan con otras empresas lo hacen en esos ámbitos. Además, debe tenerse en cuenta que hay muchos tipos distintos de cercanía relacionada con la innovación, desde la más próxima, en la propia oficina, en que se producen encuentros cara a cara con frecuencia, a la de las grandes concentraciones innovadoras, con sus ventajas competitivas. La relación entre territorio e innovación varía según la escala de análisis. Por otro lado, ha habido un gran número de enfoques teóricos diferentes sobre la vinculación entre territorio e innovación. González ${ }^{33}$ menciona cinco: medios innovadores, sistemas territoriales de innovación, nuevos espacios industriales, dinámicas de proximidad y regiones inteligentes. Además, la relevancia de la

\footnotetext{
${ }^{26}$ Foster, 2002.

${ }^{27}$ Trompenaards y Hampden-Turner, 2010.

${ }^{28}$ Koschatzky y Sternberg, 2000; Lavía y et al., 2010; Morgan, 2004; Vespagen y Schoenmakers, 2004; Drejer y Vinding, 2005; Koch y Stahlecker 2006; Inkinen y Suorsa, 2010; Oerlemans y Meeus, 2005; Torre y Rallet, 2005.

${ }^{29}$ Drejer y Vinding, 2005, citando a Feldman, 1994.

${ }^{30}$ Tödtling y Kaufman , 2002.

${ }^{31}$ Lavía et al., 2010 y Inkinen y Suorsa, 2010.

${ }^{32}$ Lavía et al., 2010.

${ }^{33}$ González, 2006.
} 
proximidad no es uniforme, sino que varía de un territorio a otro. Koschatzky y Sternberg ${ }^{34}$ estudian 10 regiones europeas y concluyen que la importancia de la proximidad para la innovación no es la misma en todas ellas. El resultado es que los territorios no son tan homogéneos como a veces se ha pensado, algo que también se había observado respecto a otros aspectos, como por ejemplo la terciarización española ${ }^{35}$.

En la práctica el territorio sigue teniendo una gran influencia en las decisiones de localización de las empresas y los trabajadores de sectores innovadores. Además, ya no se concibe como mero soporte o escenario, sino que por sí mismo es capaz de crear un entorno que propicia el desarrollo ${ }^{36}$. Según Crescenzi y Rodríguez-Pose ${ }^{37}$ hay factores que son un buen indicador de la innovación local, como la concentración de actividades de investigación y desarrollo, pero los factores socioeconómicos locales y otras características geográficas (sobre todo las economías de aglomeración) juegan también un papel importante. Y dentro de un área metropolita la innovación depende de la accesibilidad de la empresa al centro económico ${ }^{38}$.

Ménde ${ }^{39}$ señala que, a pesar de que las actividades y los profesionales del conocimiento trabajan mayoritariamente con recursos inmateriales y en teoría muy móviles, como la información y el conocimiento, el territorio está muy lejos de ser plano a la hora de que las empresas e instituciones decidan su ubicación. Las sociedades y los profesionales no son tan libres de las restricciones espaciales como las tecnologías supuestamente les permitirían. No siempre es una cuestión de que la cercanía física sea en sí misma fundamental, sino que en ella se encuentra lo importante. Por ejemplo, Jordá y Ruiz ${ }^{40}$, dicen que en un contrato de compraventa de tecnología lo que importa más es que haya proximidad cultural, tecnológica y organizativa, siendo la cercanía física menos relevante. Pero lógicamente, dónde con frecuencia suele haber más proximidad cultural, tecnológica y organizativa es en el territorio en que se está ubicado, sobre todo cuando ocupa una posición más elevada en la jerarquía urbana. Jordá, y Ruiz ${ }^{41}$, refiriéndose a Andalucía concluyen que "las economías externas y de aglomeración (componente territorial) condicionan la especialización sectorial y otras características estructurales (tamaño, capital, etc.) de cada ámbito, y consecuentemente el comportamiento innovador de las empresas que en él se ubican... En las aglomeraciones urbanas donde las economías externas son elevadas, se concentran las empresas de sectores intensivos en capital y conocimiento". Comentan que en las aglomeraciones urbanas también se encuentran las empresas más internacionalizadas y que más invierten en investigación y desarrollo. En la misma línea, para Corsatea y Jayet ${ }^{42}$, en Francia la concentración de actividades económicas y de investigación y desarrollo resultan esenciales para ser regiones innovadoras.

Otra perspectiva es la de las desigualdades que genera la relación entre innovación y territorio, puesto que se producen nuevos desequilibrios territoriales al resultar ganadores unos territorios

\footnotetext{
${ }^{34}$ Koschatzky y Sternberg, 2000.

${ }^{35}$ Gámir, A. et al., 1989.

${ }^{36}$ Caravaca et al., 2003.

${ }^{37}$ Crescenzi y Rodríguez-Pose, 2013.

${ }^{38}$ Dautel y Walter, 2014.

${ }^{39}$ Méndez, 2012.

40 Jordá y Ruiz, 2012.

${ }^{41}$ Jordá, y Ruiz, 2009.

${ }^{42}$ Corsatea y Jayet, 2014.
} 
frente a otros, que quedan en una posición marginal, o incluso excluidos del sistema ${ }^{43}$. Asimismo, los territorios más innovadores no siempre provocan una buena calidad de vida a sus habitantes. A veces generan una desigualdad socio-espacial tan aguda que por ejemplo "amenaza la continuidad del crecimiento local y regional en Silicon Valley e Irlanda"44. La aproximación a la innovación tecnológica no debe ser meramente técnica, sino también social ${ }^{45}$. El modelo de desarrollo no se debe basar solo en la innovación y el crecimiento económico, sino que debe integrar el bienestar social, la sostenibilidad ambiental y la reducción de los desequilibrios territoriales $^{46}$. Con el esfuerzo que conlleva lograr que las empresas de un determinado territorio sean innovadoras, no tendría sentido que de antemano no se adoptasen todas las medidas posibles para que el desarrollo resultante no sea beneficioso únicamente desde el punto de vista económico, sino también del social y medioambiental. Bosque ${ }^{47}$ dice que el objetivo principal y definitivo debe ser buscar la "sustentabilidad", la "habitabilidad" y la "¿felicidad?”.

Para lograr que las empresas de un territorio sean innovadoras intervienen numerosos factores. González $^{48}$ resalta que desde los años ochenta y noventa del pasado siglo un gran número de investigadores sostienen que la innovación empresarial está condicionada, no únicamente por las capacidades individuales de las empresas, sino por toda la red de agentes socioeconómicos e institucionales, así como por las políticas, los recursos y las iniciativas de su entorno. RodríguezPose y Comptour ${ }^{49}$ descubrieron que la mejor manera para generar innovación y promover un mayor crecimiento económico en un territorio consiste en que sus habitantes tengan un alto nivel de formación, es decir, de factores como tener unos buenos niveles de educación o una mano de obra con conocimientos suficientes en nuevas tecnologías.

En ocasiones las políticas de las Administraciones públicas, junto con otros factores locales, pueden resultar exitosas. Un ejemplo es el distrito 22@ de Barcelona, aunque con un efecto relativamente modesto ${ }^{50}$. En un estudio sobre la economía creativa Méndez et al. ${ }^{51}$, analizan las 851 áreas urbanas españolas. Concluyen que la mayoría de ellas quedan al margen, pero que algunas logran insertarse competitivamente, sobre todo por haber aplicado políticas de promoción de determinados clusters. Debe haber una dinámica equilibrada entre promover el desarrollo económico innovador y lograr un lugar en el que merezca la pena vivir. A este respecto Méndez et al. ${ }^{52}$ proponen que parte de la política de dinamización territorial de las ciudades sea "la construcción de redes sociales e institucionales que, además de apoyar la mencionada innovación en el seno de las empresas y la economía local, realicen acciones que favorezcan la calidad de vida urbana, el combate a los procesos de marginación y exclusión que propicia la lógica del mercado, o la protección y puesta en valor del patrimonio urbano".

\footnotetext{
${ }^{43}$ Caravaca, 1998.

${ }^{44}$ Pike et. al, 2011.

${ }^{45}$ Muscar, 2000.

${ }^{46}$ Caravaca et. al., 2007.

${ }^{47}$ Bosque, 2008.

${ }^{48}$ González, 2010.

${ }^{49}$ Rodríguez-Pose y Comptour, 2012.

${ }^{50}$ Viladecans-Marsal y Arauzo-Carod, 2012.

51 Méndez et al., 2012.

52 Méndez et al., 2006.
} 
Climent $^{53}$ comenta cuáles son las actuaciones prioritarias que el sector privado y el público deben desarrollar para lograr que un territorio sea más innovador. Sostiene que deben "centrarse en invertir en capital humano, en centros de formación, laboratorios técnicos y de investigación, infraestructuras de información y demás instituciones que lleven a cabo actividades intangibles relacionadas con el conocimiento". Caravaca et al. ${ }^{54}$, proponen cuatro ámbitos para las propuestas de actuación que conformen los sistemas locales innovadores: el espacio de organización (la empresa); el espacio de la competencia (el mercado); el espacio de la cooperación (las redes) y el espacio de la sinergia (el medio). Un problema es que muchas veces la actividad pública es insuficiente, por ejemplo en Castilla y León la Administración Regional no ha podido evitar la escasez de empresas de alta y media-alta tecnología o la concentración de la innovación en unas pocas empresas ${ }^{55}$. Otro problema es que muchas de las políticas de promoción de la innovación se aplican con frecuencia de manera errónea. González ${ }^{56}$ estudia en profundidad las políticas públicas de innovación territorial. Detalla ocho grandes críticas, como el que se prima a las empresas y sectores que ya son innovadoras y que crecen en mayor medida; se utiliza en exceso la subvención, siendo además su solicitud demasiado burocrática; falta coordinación en las políticas de formación, de investigación y de empresa; etc. Para Head ${ }^{57}$ la promoción de la innovación regional tiene que ser diseñada por las administraciones en colaboración estrecha con las empresas y con la comunidad. Y tiene que ser iterativa, es decir, con constantes ajustes a la realidad cambiante. Además, no siempre las políticas públicas son las que ofrecen mejores resultados. Pallarés y Vera ${ }^{58}$ indican que el éxito o fracaso de una empresa parece depender más de la calidad y preparación de su capital humano, que de incentivos como ayudas económicas para su ubicación.

Las medidas para fomentar la innovación no deberían ser las mismas para todos los territorios. Habría que aplicar políticas de innovación inteligentes, que se adapten a las características, fortalezas y debilidades del modelo de innovación de cada región, pero que además mejoren su especialización y diversificación tecnológica ${ }^{59}$. Sin embargo, con frecuencia las políticas de promoción de la innovación son muy similares. Pascual y García ${ }^{60}$ critican la repetición de objetivos e instrumentos similares, que pueden llevar a una homogeneización contraria a la especificidad local. Lógicamente, según las circunstancias locales, hay que añadir medidas particularizadas. Por ejemplo, Aparicio et al. ${ }^{61}$, señalan el factor decisivo que la Denominación de Origen ha tenido en el medio innovador de la comarca vitivinícola de la Ribera del Duero, que se estableció en 1982, y la integración funcional de las empresas en el sistema de innovación vitivinícola auspiciado por las autoridades regionales. Esto lo diferencia de las medidas adoptadas en las ciudades, pero es muy similar a las adoptadas en otras muchas comarcas vinícolas $^{62}$. El que haya diferenciación local en las políticas para la innovación es importante, pero es muy difícil de conseguir. Aún así, cada territorio es diferente, entre otras razones porque

\footnotetext{
${ }^{53}$ Climent, 2009.

${ }^{54}$ Caravaca et al., 2005.

${ }^{55}$ Calderón et. al, 2010 y Calderón y Pascual, 2011.

${ }^{56}$ González, 2006.

${ }^{57}$ Head, 2011.

${ }^{58}$ Pallarés y Vera, 2000.

${ }^{59}$ Capello y Lenzi, 2014.

${ }^{60}$ Pascual y García, 2008.

61 Aparicio et al., 2008.

62 Alonso, 2003.
} 
sus agentes reaccionan de manera específica. Méndez et al. ${ }^{63}$ indican que "un componente explicativo fundamental de las trayectorias industriales recientes debe buscarse en claves internas y específicas para cada ciudad, vinculadas a la diversa capacidad mostrada por sus empresas y demás actores locales para enfrentar de manera creativa el nuevo marco de competencia". Capello y Lenzi ${ }^{64}$ enfatizan que "cada región tiene que ser capaz de descubrir su modelo territorial de innovación, y solo a través de la concienciación de su original y único modelo de innovación territorial puede una región esperar ser excelente en explotar la eficiencia innovadora".

El tener y hacer uso de redes de interrelación empresarial es un factor fundamental para que un territorio sea innovador. Esta es una carencia de muchos espacios, como ha comentado Salom ${ }^{65}$ para el caso de áreas de desarrollo endógeno de la Comunidad Valenciana o Calderón y Pascual $^{66}$ para los nuevos espacios productivos en Castilla y León. Drejer y Vinding ${ }^{67}$ consideran que para la innovación las relaciones locales son importantes, pero que son mejores si se combinan con relaciones externas, lo que puede servir para promover nuevas ideas y una mayor diversidad de las mismas en el territorio. Koschatzky y Sternberg ${ }^{68}$ también advierten de la importancia de establecer redes para la innovación que combinen lo local, lo regional y lo supraregional. Señalan que las empresas intensivas en conocimiento necesitan ambos tipos de redes, entre otras razones porque hay una creciente interdependencia entre las dinámicas regionales y globales. Salom ${ }^{69}$ enfatiza asimismo la importancia de las redes externas abiertas y supralocales, junto con las locales, más estrechas y que proporcionan más oportunidades para realizar múltiples asociaciones. Fisher ${ }^{70}$ matiza que importan sobre todo los niveles internacionales y regionales, pero no tanto el nacional.

\section{Analizar la relación entre innovación y distancia}

\section{La importancia de las relaciones externas}

La hipótesis 2 dice que "las relaciones externas son importantes para la innovación". Se les preguntó a los empleados de MCS “¿en qué grado considera que sus innovaciones se deben a relaciones externas a la empresa (por ejemplo con otras empresas, proveedores, clientes, universidades, organizaciones públicas u otras)?”. El 45,71 por ciento respondió que en grado medio, un 11, 43 por ciento que bajo y un 2,86 por ciento que muy bajo. Estas respuestas muestran una menor importancia de las relaciones externas de lo esperado. Tan solo un 28,57 por ciento consideraba que las relaciones externas son importantes en grado alto y un 11,43 por ciento que en muy alto. Sin embargo, estos porcentajes superan a los que respondieron que bajo o muy bajo. Analizamos si los empleados que habían manifestado ser más innovadores consideran en un mayor grado que sus innovaciones se deben a relaciones externas a la empresa.

\footnotetext{
${ }^{63}$ Méndez et al., 2008.

${ }^{64}$ Capello y Lenzi, 2013.

65 Salom, 1997.

${ }^{66}$ Calderón y Pascual, 2009.

${ }^{67}$ Drejer y Vinding, 2005.

68 Koschatzky y Sternberg, 2000.

${ }^{69}$ Salom, 2003.

${ }^{70}$ Fisher, 2001.
} 
Aplicando la prueba Chi-Cuadrado sobre independencia de proporciones, se obtuvo $\chi^{2}=13,74$ $(g l=16, p=, 62)$. Este resultado no alcanza significación estadística. De este estudio no se puede concluir que las personas que declaran generar más innovaciones contestan a la pregunta "¿en qué grado considera que sus innovaciones se deben a relaciones externas a la empresa?" de forma distinta a las personas que declaran menos.

Según algunos autores la importancia de las relaciones externas en ser innovadores es muy grande. Kourtit et al. ${ }^{71}$ consideran que el factor más importante para el éxito es obtener sinergias del conocimiento a través de la conectividad espacial y de la conexión mediante redes industriales o institucionales. Para Johnson ${ }^{72}$ los individuos más creativos tienen amplias redes sociales que se extienden fuera de sus organizaciones y que incluyen personas de diversos campos y especialidades. Cita un estudio en el que los individuos con una red diversa y horizontal eran tres veces más innovadores que los que tenían una red uniforme y vertical. Koschatky y Sternberg ${ }^{73}$ profundizan en la importancia de las redes de relaciones externas para la innovación. Para ellos las ventajas de las redes residen en lograr sinergias al tener acceso a recursos complementarios, no disponibles por sus integrantes considerados aisladamente. También piensan que son mejores si los individuos están conectados de manera más horizontal y menos jerárquica, y si se basan en la confianza mutua. Miguélez y Moreno ${ }^{74}$ encuentran que las relaciones externas son muy importantes en los procesos de innovación local, junto con la movilidad del mercado laboral. Mukherji y Silberman ${ }^{75}$ demuestran que la capacidad de una región para absorber conocimiento externo tiene un impacto positivo, estadísticamente significativo, en su capacidad de innovación.

La presencia o ausencia de otros factores puede hacer que las interrelaciones sean poco eficaces. Fritsch y $\mathrm{Graf}^{76}$ comparan cuatro sistemas de innovación regional de Alemania del Este y del Oeste. Los dos del Este tenían un mayor grado de interacciones, pero lograban menos patentes e innovaciones que los de Oeste. Esta contradicción la explican porque en el Este han sufridos problemas económicos por la reunificación, porque son catedrales en el desierto que carecen de un buen entorno regional con el que interactuar y porque no es el nivel, sino la calidad de la cooperación lo que más influye.

\section{La importancia de unas relaciones más internacionales}

Se les preguntó “¿en qué grado considera que usted sería más innovador si sus relaciones para la innovación se hiciesen más internacionales?” Hubo 35 respuestas. El 40 por ciento consideraba que en un grado alto, el 37,14 por ciento que muy alto y el 22,86 por ciento que medio. No hubo ninguna respuesta para grado bajo o muy bajo. Analizamos si las personas que se declaran más innovadoras consideran que serían más innovadoras si sus relaciones para la innovación se hiciesen más internacionales. Aplicando la prueba Chi-Cuadrado sobre independencia de

\footnotetext{
${ }^{71}$ Kourtit et al., 2011.

72 Johnson, 2011.

${ }^{73}$ Koschatzky y Sternberg, 2000.

${ }^{74}$ Miguélez y Moreno, 2013.

${ }^{75}$ Mukherji y Silberman, 2013.

${ }^{76}$ Fritsch y Graf, 2011.
} 
proporciones, se obtuvo un valor de $\chi^{2}=6,267(g l=8, p=, 61)$. Este resultado no alcanza significación estadística, y por tanto no se concluye que las personas que declaran generar más innovaciones contesten a esta pregunta de forma distinta a las personas que declaran menos.

La importancia de las conexiones internacionales ha sido estudiada, entre otros, por Simmie ${ }^{77}$. Encontró que en el Reino Unido las empresas más innovadoras eran las que utilizan más las fuentes externas de conocimiento o de información. En cualquier caso las relaciones internacionales para la innovación son difíciles, incluso en territorios contiguos. Por ejemplo, Koschatzky y Sternberg ${ }^{78}$ observan que la cooperación para la innovación es muy escasa entre las empresas e instituciones de las regiones de Alsacia (Francia) y Baden (Alemania), que hacen frontera. Con Internet las distancias geográficas son menos problemáticas que las barreras idiomáticas y culturales. En cualquier caso, la cercanía sigue jugando un papel primordial, porque en general la intensidad de la comunicación a distancia es mayor entre las regiones vecinas, mostrando que la proximidad influye en la utilización del ciberespacio ${ }^{79}$.

Koch y Stahlecker ${ }^{80}$ investigaron los servicios empresariales intensivos en conocimiento en Bremen, Múnich y Stuttgart. Concluyeron que la proximidad geográfica a los clientes y suministradores parecía que jugaba un papel esencial, sobre todo en las primeras etapas. Asimismo encontraron que para estas empresas, durante su fundación e inicios, la proximidad territorial importaba más que en las empresas industriales. Pero para un desarrollo duradero y a largo plazo de los servicios empresariales intensivos en conocimiento, las relaciones regionales no eran una condición suficiente. Para progresar era importante una expansiva orientación hacia el exterior. Sin embargo, la mayoría de estas empresas no parecían lo suficientemente integrados en los sistemas de innovación suprarregionales.

Las ventajas que para la innovación tienen, por una parte la proximidad y, por otra la internacionalización, han sido estudiadas por De Bruijn ${ }^{81}$, que concluye que la segunda es más relevante. Analiza la innovación en Holanda y encuentra que hay resultados innovadores mayores con socios internacionales que con socios regionales. Las conexiones internacionales parecen estar muy relacionadas con la innovación, mientras que parece débil la relación entre proximidad regional e innovación. El autor advierte que algunas sinergias de proximidad regional pueden ser beneficiosas para el proceso de innovación de algunas empresas y sectores. La proximidad puede no ser en general un elemento importante para la innovación, pero en algunos casos sí lo es.

Fitjar y Rodríguez-Pose ${ }^{82}$ han estudiado las variables que influyen en las decisiones de las empresas para colaborar con socios a distintas escalas espaciales. Para tener más relaciones internacional los factores esenciales eran la educación y la apertura mental de los directivos.

\footnotetext{
${ }^{77}$ Simmie, 2003.

${ }^{78}$ Koschatzky y Sternberg, 2000.

${ }^{79}$ Tranos y Nijkamp, 2013.

${ }^{80}$ Koch y Stahlecker, 2006.

${ }^{81}$ De Bruijn, 2004.

${ }^{82}$ Fitjar y Rodríguez-Pose, 2014.
} 


\section{El lugar de las relaciones con otras empresas}

La hipótesis 3 sostiene que "los profesionales que pueden trabajar más desapegados del territorio, como los empleados de MCS, aprovechan esta oportunidad y lo hacen a distancias largas". Está subdividida en dos. La hipótesis 3a dice que "las relaciones con otras empresas son frecuentes y se producen sobre todo fuera de España". Se planteó la pregunta "señale hasta dos ámbitos geográficos a los que pertenecen otras empresas con las que usted tiene relaciones que le ayudan a ser más innovador". Hubo 35 respuestas. De ellas se desprende que las relaciones con otras empresas sí son frecuentes. Solo un 14, 29 por ciento de los que responden manifiestan que no tienen relaciones con otras empresas que le ayudan a ser más innovador. Esto es coherente con los que, en la pregunta sobre la importancia de las relaciones externas, manifestaron que son importantes en grado bajo o muy bajo. El bloque de respuestas más importantes es para las opciones de fuera de España. En primer lugar aparece la Unión Europea, con un 48,57 por ciento de los que seleccionan esta opción y, en tercer lugar, otras zonas desarrollas del mundo no hispanoparlantes, con un 42,86 por ciento.

\section{Cuadro 4 \\ Grupos sobre la ubicación de las empresas que le ayudan a ser más innovadores}

$\begin{array}{lc}\text { Respuesta } & \text { Grupo } \\ \text { - No tengo relaciones con otras empresas que me } & \\ \text { ayudan a ser más innovador } & 1 \\ \text { - Mismo municipio o municipios cercanos } & \\ \text { - Misma Comunidad Autónoma } & 2 \\ \text { - España } & \\ \text { - Naciones hispanoparlantes } & \\ \text { - Unión Europea } & \\ \text { - Otras zonas desarrolladas del mundo no } & \\ \text { hispanoparlantes } & \\ \text { - Otras zonas en vías de desarrollo del mundo no } \\ \text { hispanoparlantes }\end{array}$

El alto nivel de inglés se manifiesta aquí también y de hecho solo un 5,71 por ciento selecciona naciones hispanoparlantes. Asimismo, las relaciones son escasas con las naciones en vías de desarrollo, según se desprende de la anterior respuesta y de que únicamente un 5,71 por ciento selecciona otras zonas en vías de desarrollo del mundo no hispanoparlantes. Sin embargo, las relaciones dentro de España también son importantes, ya que aparece en segundo lugar por número de respuestas. Un 45,71 por ciento de los que responden seleccionan esta opción. Con los ámbitos geográficos más cercanos la relación es menor: un 8,57 por ciento de las respuestas son para la misma Comunidad Autónoma y un 5,71 por ciento para el mismo municipio o municipios cercanos.

Respecto a la hipótesis 3a, se aprecia una mayor relación con empresas de fuera de España, pero el territorio nacional sigue siendo muy relevante. Realmente sorprende que la relación con 
empresas exteriores sea tan fuerte, puesto que MCS tiene jurisdicción para trabajar en territorio español, por lo que su grado de colaboración con otras empresas está limitado a socias tecnológicas locales y a socios de Microsoft a nivel internacional. Analizamos si los empleados que habían manifestado ser más innovadores tenían relaciones con empresas ubicadas a mayor distancia. Aplicando la prueba Chi-Cuadrado sobre independencia de proporciones, se obtuvo un valor de $\chi^{2}=12,03(g l=12, p=, 44)$. Este resultado no alcanza significación estadística, y por tanto no se puede concluir que las personas que declaran generar más innovaciones contesten a esta pregunta de forma distinta a (pertenezcan a un grupo distinto que) las personas que declaran menos. La hipótesis 6, que sostiene que los que innovan más colaboran con empresas ubicadas a distancia mayores, no encuentra corroboración estadísticamente significativa.

Asimismo, analizamos la hipótesis 8, que sostiene que los empleados que dominan en mayor medida el inglés (nivel alto o muy alto) trabajan más con empresas de territorios no hispanoparlantes, que los que tienen un nivel medio o inferior de inglés. Para analizarlo se dividió a los empleados en tres grupos, de la manera que muestra el cuadro 4.

Para incluir a un empleado en un grupo, bastó que contestara al menos una de las opciones que dan acceso a ese grupo. Se les incluyó en el grupo más alto posible. Ejemplo: si contestó "mismo municipio" y "Unión Europea", se le consideró del grupo 3. Los empleados quedaron distribuidos como se muestra en el cuadro 5.

\begin{tabular}{|c|c|c|c|c|}
\hline \multirow[b]{2}{*}{ Grupo } & \multicolumn{3}{|c|}{$\begin{array}{l}\text { Cuadro } 5 \\
\text { bre la ubicación de las empresas } \\
\text { udan a ser más innovadores }\end{array}$} & \multirow{2}{*}{$\begin{array}{l}\text { S } \\
\text { Porcentaje } \\
\text { acumulado }\end{array}$} \\
\hline & Frecuencia & Porcentaje & $\begin{array}{l}\text { Porcentaje } \\
\text { válido }\end{array}$ & \\
\hline $\begin{array}{l}\text { Sin relación con otras } \\
\text { empresas }\end{array}$ & 6 & 16.7 & 16.7 & 16.7 \\
\hline $\begin{array}{l}\text { Relaciones con } \\
\text { empresas } \\
\text { hispanoparlantes }\end{array}$ & 4 & 11.1 & 11.1 & 27.8 \\
\hline $\begin{array}{l}\text { Relaciones con } \\
\text { empresas no } \\
\text { hispanoparlantes }\end{array}$ & 26 & 72.2 & 72.2 & 100.0 \\
\hline Total & 36 & 100.0 & 100.0 & \\
\hline
\end{tabular}

Aplicando la prueba Chi-Cuadrado sobre independencia de proporciones, se obtuvo un valor de $\chi^{2}=4,702(g l=4, p=, 319)$. Este resultado no alcanza significación estadística. No se puede concluir que los distintos grupos manifiesten un nivel diferente de inglés. 


\section{La distancia en las relaciones con las universidades}

La hipótesis $3 \mathrm{~b}$ dice que "las relaciones con universidades son frecuentes y se producen sobre todo fuera de España". Se les indicó "señale hasta dos ámbitos geográficos a los que pertenecen universidades o centros de investigación con las que usted tiene relaciones que le ayudan a ser más innovador". Hubo 34 respuestas. Un 55,88 por ciento de los que seleccionan esta opción manifiestan que no tienen relaciones con otras universidades o centros de investigación, por lo que la hipótesis no parece cierta en lo relativo a la frecuencia. Sin embargo, la dirección de la empresa señala que hay otros departamentos que tienen una mayor relación con la Universidad, colaborando en alto grado en proyectos de innovación con ellas, especialmente el Área de Evangelización y en los Centros de Innovación que tiene Microsoft en varias ciudades españolas.

Algunas investigaciones han encontrado que las PYME cooperan poco con los intermediariarios de la innovación, especialmente con las universidades ${ }^{83}$. La cooperación es incluso menor con las universidades extranjeras ${ }^{84}$. En esta falta de cooperación cabe suponer que las universidades también tienen parte de responsabilidad, sobre todo al no ser capaces de hacer una oferta los suficientemente atractiva para las empresas. En una encuesta a PYME industriales de Guipúzcoa, realizada por Lavía et al. ${ }^{85}$, de los componentes del entorno regional relacionados con la investigación y desarrollo, las universidades obtienen una puntuación de 2,92 sobre 5, es decir, están por encima del aprobado.

\begin{tabular}{|c|c|c|c|c|}
\hline \multirow[b]{2}{*}{ Grupo } & \multicolumn{3}{|c|}{$\begin{array}{l}\text { Cuadro } 6 \\
\text { los empleados más innovadores } \\
\text { ación de las universidades }\end{array}$} & \multirow[b]{2}{*}{$\begin{array}{l}\text { Porcentaje } \\
\text { acumulado }\end{array}$} \\
\hline & Frecuencia & Porcentaje & $\begin{array}{l}\text { Porcentaje } \\
\text { válido }\end{array}$ & \\
\hline $\begin{array}{l}\text { Sin relación con otras } \\
\text { universidades }\end{array}$ & 21 & 58.3 & 58.3 & 58.3 \\
\hline $\begin{array}{l}\text { Relaciones con universidades } \\
\text { hispanoparlantes }\end{array}$ & 8 & 22.2 & 22.2 & 80.6 \\
\hline $\begin{array}{l}\text { Relaciones con universidades no } \\
\text { hispanoparlantes }\end{array}$ & 7 & 19.4 & 19.4 & 100.0 \\
\hline Total & 36 & 100.0 & 100.0 & \\
\hline
\end{tabular}

Sin embargo, son el penúltimo elemento menos valorado. Se situaron detrás de la red de centros tecnológicos $(3,25)$, el acceso a proveedores $(3,18)$, las infraestructuras físicas $(3,07)$, los centros de formación profesional $(3,03)$, el acceso a clientes $(2,99)$ y la disponibilidad de mano de obra cualificada $(2,93)$. Solo el acceso a programas y ayudas públicas $(2,90)$ obtiene una puntuación peor. Destacan que las PYME de mayor tamaño valoran más positivamente a las universidades.

La hipótesis $3 \mathrm{~b}$ tampoco parece sostenerse respecto al ámbito geográfico. Al contrario de lo que sucedía con las empresas, en las universidades la mayor parte de las relaciones son locales. El

\footnotetext{
${ }^{83}$ Tödtling y Kaufmann, 2002; Inkinen y Suorsa, 2010.

${ }^{84}$ Inkinen y Suorsa, 2010.

${ }^{85}$ Lavía et al., 2010.
} 
20,59 por ciento de los que responden seleccionan a la misma Comunidad Autónoma, un 17,65 por ciento a España y un 5,88 por ciento al mismo municipio o municipios cercanos. Fuera de España, con pocas respuestas, destacan otras zonas desarrollas del mundo no hispanoparlantes con un 11,76 por ciento, la Unión Europea con un 8,82 por ciento, naciones hispanoparlantes con otro 8,82 por ciento y otras zonas en vías de desarrollo del mundo no hispanoparlantes, con un 2,94 por ciento. Analizamos si los empleados que habían manifestado ser más innovadores tenían relaciones con universidades a más larga distancia. Se dividió a los empleados igual que en la pregunta anterior. El cuadro 6 refleja su distribución.

Aplicando la prueba Chi-Cuadrado sobre independencia de proporciones, se obtuvo un valor de $\chi^{2}=24,34(g l=16, p=, 08)$. Este resultado no alcanza significación estadística. Por tanto no podemos concluir que las personas que declaran generar más innovaciones contesten a esta pregunta de forma distinta a las personas que declaran menos. La hipótesis 7, que sostiene que los que innovan más colaboran con universidades ubicadas a distancias mayores, no encuentra corroboración estadísticamente significativa.

\section{Las concentraciones de empresas de alta tecnología}

La hipótesis 4 sostiene que "disponer cerca de una alta concentración de empresas de alta tecnología es beneficioso para la innovación”. A los empleados de MCS se les preguntó "¿existe en su municipio o en municipios próximos una alta concentración de empresas innovadoras?". En la pregunta se sobrentiende que no se refiere a estar ubicado en un espacio innovador relativamente pequeño, como un parque tecnológico de reducidas dimensiones. La pregunta no se plantea así, porque en ese caso la posibilidad de una red de relaciones para la innovación está más limitada, por falta de masa crítica y porque pocos espacios innovadores pequeños son lo suficientemente dinamizadores. Benito del Pozo ${ }^{86}$ afirma que son escasos los parques tecnológicos que actúan como verdaderos medios innovadores. Sus objetivos son lograr concentraciones de empresas de alta o nueva tecnología, sin crear sinergias que originen redes empresariales. La misma autora unos años más tarde, mantiene y matiza la crítica. Señala que en la mayoría de los casos son una infraestructura más de apoyo a la innovación tecnológica, o un espacio con empresas innovadoras, pero sin capacidad para generar redes de innovación en su entorno $^{87}$. Por ello la encuesta se refiere a estar en una ciudad que pueda tener uno o varios parques tecnológicos, así como cualquier otra concentración de empresas, universidades, centros de investigación y otras instituciones innovadoras. Hubo 34 respuestas, que mayoritariamente muestran una postura contraria a la hipótesis. El 58,82 por ciento contestó que sí existe pero no afecta a su grado de innovación y únicamente un 17,65 por ciento que sí existe y ello facilita su grado de innovación. Un 23,54 por ciento piensa que no existe. Analizamos si las personas que se declaran más innovadoras afirman que en su entorno existe una alta concentración de empresas innovadoras y, en caso de que exista, si consideran que ello favorece su innovación. Aplicando la prueba Chi-Cuadrado sobre independencia de proporciones, se obtuvo un valor de $\chi^{2}=19,77(g l=8, p<, 05)$. Este resultado sí alcanza significación estadística. Las personas que declaran generar más innovaciones contestan a esta pregunta de forma distinta a las personas que declaran menos. No obstante, la relación es difícil de interpretar.

\footnotetext{
${ }^{86}$ Benito del Pozo, 2000.

${ }^{87}$ Benito del Pozo, 2005.
} 
En el cuadro 7 se muestra cómo se agrupan las personas en función de su respuesta combinada a ambas preguntas. Se marcan en gris aquellas casillas en las que hay más personas de lo que cabría esperar si no hubiera relación entre ambas preguntas.

Como se puede observar, no hay una pauta clara de asociación entre las variables. La hipótesis 4, que sostiene que el disponer cerca de una alta concentración de empresas de alta tecnología es beneficioso para la innovación, no encuentra corroboración estadísticamente significativa.

\section{Cuadro 7}

\section{Relación entre concentración de empresas innovadoras y grado de innovación}

Indique el número de innovaciones (mejoras relevantes de productos, procesos $u$ otras que sean o tengan potencial de ser un éxito comercial) que usted haya generado en los últimos 3 años y que considere que son pioneras a escala internacional

\begin{tabular}{|c|c|c|c|c|c|c|c|}
\hline & & 0 & $1-3$ & $3-4$ & $5-6$ & $6+$ & \\
\hline \multirow{3}{*}{$\begin{array}{l}\text { ¿Existe en su } \\
\text { municipio o en } \\
\text { municipios } \\
\text { próximos una alta } \\
\text { concentración de } \\
\text { empresas } \\
\text { innovadoras? }\end{array}$} & $\begin{array}{l}\text { Sí existe y ello facilita mi } \\
\text { grado de innovación }\end{array}$ & 1 & 3 & 0 & 2 & 0 & 6 \\
\hline & $\begin{array}{l}\text { Sí existe pero no afecta a mi } \\
\text { grado de innovación }\end{array}$ & 8 & 8 & 4 & 0 & 0 & 20 \\
\hline & No existe & 2 & 4 & 0 & 0 & 2 & 8 \\
\hline Total & & 11 & 15 & 4 & 2 & 2 & 34 \\
\hline
\end{tabular}

Fuente: elababoración propia

\section{La búsqueda de lo mejor}

La hipótesis 5 dice "se preferirá un socio ligeramente mejor, ubicado más lejos, que uno ligeramente peor, pero ubicado más cerca". Se les preguntó "¿en qué grado preferiría un socio para la innovación que sea ligeramente peor, pero ubicado en su mismo municipio (o en municipios cercanos), respecto a otro que sea ligeramente mejor, pero ubicado en una zona de España alejada de su empresa?" Esta pregunta puede permitir calibrar la importancia de la proximidad dentro de un mismo entorno lingüístico, a una distancia no demasiado larga y con un margen de mejora pequeño. Si las empresas contestan que prefieren lo ligeramente mejor lejos, sugeriría que la proximidad cuenta poco, mientras que lo contrario pasaría en caso de optar por lo ligeramente peor cerca. Hubo 35 respuestas. Para la mayoría la cercanía no compensa el que se sea ligeramente peor. Un 57,15 por ciento piensa que en grado bajo o muy bajo (un 34,29 por ciento y un 22,86 por ciento respectivamente). En grado medio, reflejando que se da una cierta importancia a la cercanía, contesta el 28,57 por ciento. En grado alto solo un 14,29 por ciento y 
no hay ninguna respuesta en muy alto. El preferir lo lejano que es mejor a lo peor más cercano parece la mejor opción. Cabe suponer que ofrecerá un mejor resultado, según se desprende de las propias respuestas y de lo comentado en otros lugares de este trabajo. Analizamos en qué medida los empleados que se declaran más innovadores preferirían un socio para la innovación que sea ligeramente peor, pero ubicado en su mismo municipio (o en municipios cercanos), respecto a otro que sea ligeramente mejor, pero ubicado en una zona de España alejada de su empresa. Aplicando la prueba Chi-Cuadrado sobre independencia de proporciones, se obtuvo un valor de $\chi^{2}=10,276(g l=12, p=, 592)$. Este resultado no alcanza significación estadística, y por tanto no se puede concluir que las personas que declaran generar más innovaciones contesten a esta pregunta de forma distinta a las personas que declaran menos.

Si las relaciones para la innovación se enfocan hacia interconectarse con los profesionales mejores, estén a la distancia que estén, entonces la lejanía primaría sobre la cercanía porque normalmente los mejores estarán lejos y dispersos. Cabe suponer que la mayoría de las veces las mejores colaboraciones posibles no son locales, sino muy lejanas, tanto de uno mismo como entre sí. Ser capaz de relacionarse con lugares distantes permite aprovechar globalmente las ventajas comparativas internacionales de cada territorio.

Posiblemente el Silicon Valley sea el mejor exponente de un espacio en que los mejores están cerca y, además, en gran abundancia. Pocos lugares del mundo disfrutan simultáneamente de las sinergias para la innovación que produce, por una parte la proximidad de los mejores con, por otra, tener redes importantes de relaciones internacionales con lo mejor lejano. El resto de territorios y empresas en general carecen de un espacio local innovador con los mejores. Sin embargo, está a su alcance el buscar lo mejor en lugares lejanos. Si esto es cierto, las empresas y las administraciones públicas deben fomentar en mayor medida las relaciones para la innovación a larga distancia, dándolas preferencia respecto a las locales, entre otras razones porque ello reforzará el dinamismo innovador local.

\section{El teletrabajo y la proximidad cara a cara}

Si el teletrabajo se realiza a tiempo parcial, puede aunar muchas de las ventajas de la proximidad y de la distancia. Algunos autores han resaltado la importancia de estar muy cerca. De acuerdo

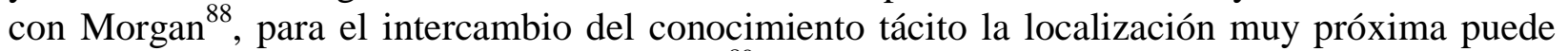
ser un factor importante. Oerlemans y Meeus ${ }^{89}$ opinan lo mismo, aunque también mencionan que en ciertos casos puede transmitirse a distancia. Estar muy cerca puede ser una ventaja para el intercambio de conocimiento. Las reuniones cara a cara se pueden celebrar con más frecuencia y de manera más eficiente que los encuentros en línea. Esto es particularmente cierto cuando lo que se negocia tiene que cambiarse en función de los gestos y del lenguaje corporal de otras personas. Con mayor proximidad, pueden suceder más fácilmente y frecuentemente situaciones inesperadas de interés. Puede haber más encuentros no programados con personas que se cruzan por casualidad, por ejemplo a la hora de comer. La confianza mutua se puede conseguir mejor.

\footnotetext{
${ }^{88}$ Morgan, 2004.

${ }^{89}$ Oerlemans y Meeus, 2005.
} 
Koschatzky y Sternberg ${ }^{90}$ afirman que la proximidad geográfica, social y cultural favorecen esos procesos que demandan los contactos interpersonales y la confianza.

Hacia 1994 Microsoft no era partidaria del teletrabajo. Sin embargo, estaba diciendo a sus clientes que se conectaran, que con sus ordenadores, su software y las telecomunicaciones se podía trabajar desde casa y vencer las distancias. Esto se ha denominado personalidad dividida. La empresa alegaba que el teletrabajo no estaba explícitamente prohibido, pero que muchos grupos de programación tenían trabajos que requerían interacciones frecuentes entre ellos en los pasillos, en las oficinas, en las salas de conferencias o en las cafeterías. El proceso para obtener permiso para teletrabajar era complejo. El empleado tenía que demostrar, entre otros requisitos, que no impedía los objetivos de grupo, que no iba a aislarse del equipo, que iba a poder ayudar a sus compañeros en sus tareas y que no iba a suponer ningún coste adicional para la empresa ${ }^{91}$.

La situación parece haber cambiado, al menos en algunas filiales, siendo de destacar Microsoft Holanda. Entre 2007 y 2010 la introducción de nuevas maneras de trabajar desde fuera de la oficina incrementaron la productividad percibida en un 2,5 por ciento, además de mejorar el equilibrio entre trabajo y vida privada, así como suponer beneficios medioambientales. Por encima del 50 por ciento de sus empleados trabajaban desde casa más de dos días a la semana ${ }^{92}$. Para la empresa tiene las ventajas adicionales de hacerse más atractiva para los empleados y, con ello, poder atraer mejor a los profesionales más cualificados. Respecto al impacto territorial de teletrabajar hay que tener en cuenta que afecta no solo al espacio productivo, sino, como señala Sánchez ${ }^{93}$, también a los espacios cotidianos (de consumo, educativos, sanitarios, asistenciales, de ocio, etc.).

En MCS el 22,22 por ciento de los empleados encuestados teletrabajan desde su domicilio tres o más días a la semana, mientras que no lo hacen el 77,78 por ciento. Este dato puede interpretarse de forma muy distinta. Por una parte, que el teletrabajo sea relativamente minoritario en MCS puede indicar que los encuentros presenciales son importantes. Pero, desde otro punto de vista, puede considerarse que teletrabajan un número relativamente alto de empleados, teniendo en cuenta que las razones de no hacerlo no sería únicamente el que la proximidad ayuda a la productividad, sino también otras como el que se ejerce un mayor control sobre los trabajadores o el que a éstos les puede no gustar. Trabajar en la oficina con los compañeros tiene las ventajas de la proximidad. Si un 22,22 por ciento decide no aprovecharlas y la empresa lo permite, significa que no son tan importantes o que a distancia también se puede disfrutar de ellas gracias a las nuevas tecnologías. Por otro lado, la dirección de la empresa manifiesta que no teletrabajar desde casa no significa que se trabaja desde la oficina. Al contrario, casi todo el tiempo los empleados de MCS trabajan desde donde están sus clientes, por lo que la mayoría teletrabaja cerca del 90 por ciento de su tiempo. Esto no es nada representativo del resto de los negocios de Microsoft en España, según señala la dirección de la empresa. Dentro de su modelo de trabajo flexible, los empleados de la empresa en su conjunto trabajan fuera de la oficina aproximadamente un 50 por ciento del tiempo (desde el domicilio o desde cualquier tercer lugar). Sus oficinas son "lugares de encuentro", no un lugar de trabajo tradicional. Analizamos si los

\footnotetext{
${ }^{90}$ Koschatzky y Sternberg, 2000.

${ }^{91}$ Stross, R., 1997.

92 Heck, E. V. et al., 2012.

93 Sánchez, 1991.
} 
empleados que se declaran más innovadoras son teletrabajadores. Aplicando la prueba ChiCuadrado sobre independencia de proporciones, se obtuvo un valor de $\chi^{2}=1,971(\mathrm{gl}=4, p$ $=, 741)$. Este resultado no alcanza significación estadística. No se puede concluir que las personas que teletrabajan declaran generar menos innovaciones que las que no teletrabajan. La hipótesis 10 de que los que teletrabajan innovan menos que los que no teletrabajan no tiene corroboración estadísticamente significativa.

Otra perspectiva sobre las ventajas de trabajar en la misma oficina, la proporciona el contraste entre las preferencias de los trabajadores cuando tienen que mudarse y lo que opinan los técnicos que planifican el traslado. Los diferentes grupos de programación de Microsoft preferían estar juntos en el mismo edificio, pero el crecimiento de empleados de la empresa fue tan vertiginoso (3 en 1975, 910 en 1985, 17.801 en 1995, etc.) que esto resultó imposible. Los gestores de espacios de la empresa respondían que según sus estudios la comunicación cara a cara solo se hacía unos pocos metros alrededor de cada oficina. Sus análisis concluyen que una gran proximidad no parecía favorecer la fertilización diaria de ideas ${ }^{94}$.

En contra de esta idea Yahoo!, en el año 2013, terminó su política de permitir teletrabajar a sus empleados desde su domicilio. La principal razón era que la proximidad favorecía la creatividad. Marissa Mayer, su Directora General, considera que la comunicación y la colaboración son importantes, para lo que es bueno trabajar codo con codo. Piensa que algunas de las mejores decisiones e ideas vienen de los encuentros en los pasillos o en las cafeterías, de conocer gente nueva y de reuniones improvisadas ${ }^{95}$. Es fundamental trabajar en equipo y ello posiblemente se puede hacer mejor si se producen encuentros frecuentes cara a cara. Johnson ${ }^{96}$ señala el caso concreto de una empresa de biología molecular en la que se descubrió que las ideas más importantes emergían en las reuniones periódicas en los laboratorios, donde aproximadamente una docena de investigadores se encontraban y, de manera informal, presentaban y discutían su trabajo más reciente. Sorprende que a pesar de toda la tecnología avanzada de la que dispone la empresa, lo que resultase más productivo para la innovación fuese un grupo de personas alrededor de una mesa. Las preguntas y comentarios de los compañeros fuerzan a los investigadores a reflexionar, les hace ver errores y les proporcionan nuevas ideas. Respecto al teletrabajo desde el domicilio, lo que parece recomendable es que sea tan solo durante unos días a la semana. De esta manera se puede remediar la falta de contacto personal y directo con el resto del equipo.

Los trabajadores encuestados de MCS piensan que el teletrabajo que ellos ejercen no afecta negativamente en su innovación. Un 36,11 por ciento considera que el teletrabajo puede repercutir desfavorablemente en la innovación en un grado muy bajo y un 44,44 por ciento que en un grado bajo. En grado medio contestan el 13,89 por ciento, en alto el 5,56 por ciento y muy alto el 0 por ciento. Analizamos si los que declaran ser más innovadores consideran que teletrabajar puede repercutir desfavorablemente en la innovación de la empresa. Aplicando la prueba Chi-Cuadrado sobre independencia de proporciones, se obtuvo un valor de $\chi^{2}=15,566$ $(g l=12, p=, 212)$. Este resultado no alcanza significación estadística.

\footnotetext{
${ }^{94}$ Stross, R., 1997.

95 Martín, J., 2013.

96 Johnson, 2011.
} 
Aunque no necesariamente tres o más días a la semana, ni desde el domicilio, el teletrabajo, al menos de manera parcial, está muy extendido en las empresas. La encuesta de uso de TIC y comercio electrónico en las empresas 2011-12 manifiesta que el 60,3 por ciento de ellas disponían de empleados que trabajan fuera de los locales de la empresa de forma regular (al menos media jornada semanal) y se conectaban a los sistemas TIC de la empresa mediante redes telemáticas externas ${ }^{97}$.

\section{Conclusiones}

Del análisis crítico de la bibliografía, de la encuesta y de las reflexiones propias parecen desprenderse las siguientes conclusiones:

1. Microsoft tal vez haya sido la empresa más innovadora de todos los tiempos o, al menos una de ellas. Sin embargo, últimamente ha perdido posiciones respecto a otras, sobre todo Apple y Google. En cualquier caso, el futuro está abierto, no solo respecto a estas tres empresas, sino también otras, que podrían ser veteranas que se fortaleciesen, como IBM o Amazon, u otras relativamente recién llegadas que irrumpan con fuerza, como Facebook.

2. La importancia de la proximidad en la innovación varía mucho en diferentes empresas, sectores, tipos de relación y territorios. Hay casos en que es muy relevante, pero en otros no lo parece tanto.

3. Parece decisiva para obtener mejores resultados la conexión mediante sistemas telemáticos con las mejores fuentes de innovación posibles, que normalmente están lejos y dispersas. Las empresas y las administraciones públicas deben fomentar en mayor medida las relaciones para la innovación a larga distancia, dándolas preferencia respecto a las locales.

4. Combinar para la innovación las relaciones externas lejanas con las locales próximas, parece una excelente fórmula para obtener mejores resultados. Las empresas y sus empleados deben estar conectadas a redes de relaciones que vayan de lo global a lo local, a ser posible por ese orden. En lo global encuentran lo mejor, pero con dificultades idiomáticas, legales y de todo tipo. Deben acceder a ello en la medida en que puedan, venciendo los problemas de que está lejano y disperso. Por su parte, en lo local, próximo y concentrado, se encuentra más accesible lo menos malo posible. Esta es muchas veces es la única opción factible o la más eficiente, dada su facilidad de acceso.

5. De la encuesta a los empleados del departamento de MCS de Microsoft Ibérica no se desprenden relaciones estadísticamente significativas. Sin embargo, sus datos parecen indicar que: es una empresa innovadora, sobre todo teniendo en cuenta que la encuesta se ha realizado a consultores y no a personal dedicado a la investigación y desarrollo; se ha encontrado un aspecto en el que tal vez podrían mejorar, consistente en que su consultores dispusiesen de un tiempo específico para pensar cómo ser más innovadores; mayoritariamente sus empleados piensan que serían más innovadores si sus relaciones fueran más internacionales; las relaciones con otras empresas que les ayudan a ser más innovadores se producen sobre todo fuera de España, siendo de destacar la Unión Europea y otras zonas desarrollas del mundo no hispanoparlantes; en un 56 por ciento no tienen relaciones que les ayuden a ser más innovadores con las universidades o centros de investigación, aunque sí lo tienen otros departamentos de la empresa; mayoritariamente consideran que el tener próximas una alta concentración de empresas innovadoras no contribuye

${ }^{97}$ INE, 2013. 
a incrementar su grado de innovación; la mayoría prefiere un socio para la innovación lejano que sea mejor, a otro ligeramente peor pero más cercano; hay una relativamente importante presencia de teletrabajadores, puesto que el 22,22 por ciento de los empleados encuestados teletrabajan desde su domicilio tres o más días a la semana. Además teletrabajan fuera de la oficina cerca del 90 por ciento de su tiempo, casi siempre en las oficinas de los clientes. Pero esto es específico de MCS y no es representativo del conjunto de Microsoft Ibérica; y mayoritariamente piensan que el teletrabajo no influye desfavorablemente en la innovación.

\section{Bibliografía}

ACTUALIDAD ECONÓMICA. 5000 mayores empresas de España. Iberinform. [Excel]. 2012. Recurso de pago. <http://www.ranking5000.com/>. [10 de noviembre de 2012].

ALONSO, J. L. Redes y procesos de innovación en las comarcas vinícolas de Castilla y León: el ejemplo de la D. O. Bierzo. Boletín de la Asociación de Geógrafos Españoles, 2003, nº 36, p. 4360.

ANDREW, J. P. y SIRKIN, H. L. Explota tu innovación. Madrid: LID/Harvard Business School Press, 2008.

APARICIO, J.; SÁNCHEZ, J. L.; ALONSO, J. L. y RODERO, V. La Ribera del Duero, Geografía de un medio innovador en torno a la vitivinicultura. Scripta Nova, Revista Electrónica de Geografía y Ciencias Sociales. [En línea]. Barcelona: Universidad de Barcelona, 15 de noviembre de 2008. Vol. XII, no 277. [22 de mayo de 2013]. ISBN: 84-475-2488-4.

ARTHUR, C. Digital wars: Apple, Google, Microsoft and the battle for the Internet. Londres: Kogan Page, 2012.

BANK, D. Breaking Windows: how Bill Gates fumbled the future of Microsoft, Nueva York: Free Press, 2001.

BARR, A. Proudly serving my corporate masters: what I learned in ten years as a Microsoft programmer, Bloomington (EE.UU.): Writers Club Press, 2000.

BENITO DEL POZO, P. El fenómeno de los "tecnopolos" en España. Los casos comparados de Asturias y Castilla y León. En AA.VV.: Lecturas geográficas. Homenaje a José Estébanez. Madrid: Universidad Complutense, vol. 2, 2000, p. 971-981.

BENITO DEL POZO, P. Pautas actuales de la relación entre industria y ciudad. Ería, 2005, n ${ }^{\circ}$ 66 , p. 57-70.

BHANJ, Z. Transnational Private Authority in Education Policy in Jordan and South Africa: The Case of Microsoft Corporation. Comparative Education Review, 2012, vol. 56, nº 2, p. 300319. 
BOSQUE, J. Hacia un nuevo modelo de ciudad. Vegueta, 2008, nº 10, p. 59-78.

BVD/ID\&B (Bureau Van Dijk/Informa D \& B) [En línea]. 2013. Recurso de pago. SABI (Sistema de Balances Ibéricos) <https://orbis.bvdinfo.com/version2013515/Search.QuickSearch.serv?_CID=147\&context=2JR38UYKX3PPWJG>. [16 de mayo de 2013].

CALDERÓN, B.; GARCÍA, J. L. y PASCUAL, H. De la política de innovación al desarrollo territorial innovador en las ciudades medias: el caso de Aranda de Duero. Scripta Nova, Revista Electrónica de Geografía y Ciencias Sociales. [En línea]. Barcelona: Universidad de Barcelona, 20 de noviembre de 2010, vol. XIV, nº 342. [10 de abril de 2013]. ISBN: 84-475-2488-4.

CALDERÓN, B. y PASCUAL, H. Infraestructuras de soporte en los procesos de innovación regional: nuevos espacios productivos en Castilla y León. Boletín de la Asociación de Geógrafos Españoles, 2009, nº 49, p. 237-254.

CALDERÓN, B. y PASCUAL, H. Política de innovación y estrategia de desarrollo territorial en Castilla y León. Boletín de la Asociación de Geógrafos Españoles, 2011, n 55, p. 231-249.

CAPELLO, R. y LENZI, C. Spatial heterogeneity in knowledge, innovation, and economic growth nexus: conceptual reflections and empirical evidence. Journal of Regional Science, 2014, vol. 54, no 2, p. 186-214.

CAPELLO, R. y LENZI, C. Territorial patterns of innovation: a taxonomy of innovative regions in Europe. Annals of Regional Science, 2013, nº 51, p. 119-154.

CARAVACA, I. Los nuevos espacios ganadores y emergentes. EURE, Revista Latinoamericana de Estudios Urbanos Regionales, 1998, vol. 24, n 73, p. 5-30.

CARAVACA, I.; GONZÁLEZ, G. y MENDOZA, A. Indicadores de dinamismo, innovación y desarrollo. Su aplicación en ciudades pequeñas y medias de Andalucía. Boletín de la Asociación de Geógrafos Españoles, 2007, nº 43, p. 131-154

CARAVACA, I.; GONZÁLEZ, G. y SILVA, R. Innovación, redes, recursos patrimoniales y desarrollo territorial. EURE, Revista Latinoamericana de Estudios Urbanos Regionales, 2005. Vol. XXXI, nº 94, p. 5-24.

CARAVACA, I.; GONZÁLEZ, G. y SILVA, R. Redes e innovación socio-institucional en sistemas productivos locales. Boletín de la Asociación de Geógrafos Españoles, 2003, n 36, p. $103-115$.

CRESCENZI, R. y RODRÍGUEZ-POSE, A. R\&D. Socio-Economic Conditions, and Regional Innovation in the U.S. Growth and Change, 2013, vol. 44, n' 2, p. 287-320.

CLIMENT, E. Los sistemas productivos locales: de la especialización flexible a la mente invisible y la gobernanza en red. Ería, 2009, no 78-79, p. 139-153. 
CORSATEA, T. D. y JAYET, H. Spatial patterns of innovation activities in France: market's role versus public research efforts. Annals of Regional Science, 2014, nº 52, p. 739-762.

CUSUMANO, M. A. Competing on Internet time: lessons from Netscape and its battle with Microsoft. Nueva York: Touchstone, 2000.

CUSUMANO, M. A. Staying power: six enduring principles for managing strategy and innovation in an uncertain world (lessons from Microsoft, Apple, Intel, Google, Toyota and more). Oxford: Oxford University Press, 2012.

DAUTEL, V. y WALTER, O. The geography of innovation in a small metropolitan region: An intra-regional approach in Luxembourg. Papers in Regional Science, 2014, vol. 93, nº 4, p. 703726.

DE BRUIJN, P. J. M. Mapping innovation: regional dimensions of innovation and networking in the Netherlands. Tijdschrift voor Economische en Sociale Geografie, 2004, vol. 95, n 4, p. 443440.

DREJER, I. y VINDING, A. L. Searching near and far. Determinants of innovative firms' propensities to collaborate across geographical distances. Paper presented at the DRUID Tenth Anniversary Summer Conference, Copenhagen, Denmark, 2005.

EUROPA PRESS. Google supera a Microsoft en capitalización bursátil. El Mundo.es, [En línea] http://www.elmundo.es/elmundo/2012/10/01/navegante/1349116254.html , 1 de 10 de 2012. [16 de abril de 2013].

ELBOJ, C.; PULIDO, M. A. y WELICALA, T. Las tecnologías de la innovación y la comunicación en la salida del aislamiento rural. El caso de Ariño. Scripta Nova, Revista Electrónica de Geografía y Ciencias Sociales. [En línea]. Barcelona: Universidad de Barcelona, 20 de enero de 2013, vol. XVII, no 427 (2), [26 de febrero de 2013]. ISBN: 84-475-2488-4.

FELDMAN, M. P. The Geography of innovation. Dordrecht: Kluwer Academic Publisher, 1994.

FISHER, M. Innovation, knowledge creation and systems of innovation. The Annals of Regional Science, 2001, vol. 35, n 2, p. 199-216.

FITJAR, R. D. y RODRÍGUEZ-POSE, A. The geographical dimension of innovation collaboration: networking and innovation in Norway, 2014, vol. 51, n 12, p. 2572-2595.

FOLEY, M. J. Microsoft 2.0: how Microsoft plans to stay relevant in the post-Gates era, Indeanapolis: Wiley Publishing, 2008.

FOSTER, T. R. 101 métodos para generar ideas. Cómo estimular la creatividad. Bibao: Ediciones Deusto, 2002. 
FRITSCH, M. y GRAF, H. How sub-national conditions affect regional innovation systems: The case of the two Germanys. Papers in Regional Science, 2011, vol. 90, nº 2, p. 331-354.

GÁMIR, A.; MÉNDEZ, R.; MOLINERO, T. y RAZQUÍN, J. Terciarización económica y desarrollo regional en España. Anales de Geografía de la Universidad Complutense, 1989, nº 9 , p. 123-144.

GATES, B. Camino al futuro. Madrid: Mc Graw Hill, 1995.

GONZÁLEZ, G. Innovación territorial y políticas públicas. Boletín de la Asociación de Geógrafos Españoles, 2006, nº 42, p. 121-136.

GONZÁLEZ, G. Metodología e indicadores para el análisis territorial de la innovación. Su aplicación a Andalucía. Clm.economía. Revista Económica de Castilla - La Mancha, 2010, nº 16, p. 233-256.

GPTWE (GREAT PLACE TO WORK EN ESPAÑA). Las mejores empresas para trabajar en España, <http://www.greatplacetowork.es/mejores-empresas/las-mejores-empresas-paratrabajar-en-espana/641-2012>, 2013. [7 de mayo 2013].

HEAD, B. W. Governance for sustainable regions: can government meet the innovation policy challenge? Regional Science Policy \& Practice, 2011, vol. 3, n 3, p. 219-230.

HEATH, P. y BELL, N. The changing world of home technology: a Microsoft case study. The Information Society, 2006, nº. 22, p. 251-259.

HECK, E. VAN; BAALEN, P. VAN; MEULEN, N. VAN DER y OOSTERHOUT, M. VAN. Achieving high perfomance in a mobile and green workplace. Lessons from Microsoft Netherlands. MIS Quarterly Executive, 2012, vol. 11, n 4, p. 175-188.

HEILEMANN, J. Pride before the fall: the trails of Bill Gates and the end of the Microsoft era. Darby (EE.UU.): Diane Pub Co., 2003.

ICHBIAH, D. Las cuatro vidas de Steve Jobs [1955-2011]. Madrid: LID, 2011.

INE. Encuesta de uso de TIC y comercio electrónico en las empresas 2011-2012. [En línea] <http://www.ine.es>, 2013. [19 de junio de 2013].

INKINEN, T. y SUORSA, K. Intermediaries in regional innovation systems: high-technology enterprise survey from Northern Finland. European Planning Studies, 2010, vol. 18, nº 2, p. 169187.

JOHNSON, S. Where good ideas come from. London: Penguin Books, 2011. 
JORDÁ, R. M. y RUIZ, F. Comportamiento innovador de las empresas internacionalizadas por ámbitos territoriales en Andalucía. Flujos y sistemas. Boletín de la Asociación de Geógrafos Españoles, 2009, $\mathrm{n}^{\circ}$ 50, p. 315-348.

JORDÁ, R. M. y RUIZ, F. Compra tecnológica y flujos en Andalucía. Boletín de la Asociación de Geógrafos Españoles, 2012, nº 60, p. 165-186.

KLEIN, D.; SCHMELING, J. y BLANK, P. Emerging technologies and corporate culture at Microsoft: a methodological note. Behavioral Science and the Law, 2005, n 23, p. 65-96.

KOCH, A. y STAHLECKER, T. Regional innovation systems and the foundation of knowledge intensive business services. A comparative ctudy in Bremen, Munich, and Stuttgart, Germany. European Planning Studies, 2006, vol. 14, nº 2, p.123-145.

KOSCHATZKY, K. y STERNBERG, R. R\&D cooperation in Innovation Systems-Some lessons from the European Regional Innovation Survey (ERIS). European Planning Studies, 2000, vol. 8, $\mathrm{n}^{\circ} 4$, p. 487-501.

KOURTIT, K.; NIJKAMP, P.; LOWIK, S.; VUGHT, F. V. y VULTO, P. From islands of innovation to creative hotspots. Regional Science Policy \& Practice, 2011, vol. 3, n ${ }^{\circ}$ 3, p. 145162.

LAVIA, C.; OTERO, B.; OLAZARAN, M. y ALBIZU, E. Innovación y territorio. Una encuesta a pequeñas y medianas empresas industriales. Revista Internacional de Sociología, 2010, vol. 69, $\mathrm{n}^{\circ} 2$, p. 461-486.

LEE, Z. y WOODLIFFE, L. Donor misreporting: conceptualizing social desirability bias in giving surveys. Voluntas, 2010, nº 21, p. 569-587.

MANES, S. Y ANDREWS, P. Gates. How Microsoft mogul reinvented an industry and made himself the richest man in America. Nueva York: Touchstone, 1994.

MARTIN, J. Yahoo! acaba con el trabajo en casa. El País, 28-2-2013, p. 52.

MÉNDEZ, R. Crecimiento y crisis en la región metropolitana de Madrid: significado y contradicciones de la economía del conocimiento. Revista de Geografía Norte Grande, 2012, ${ }^{\circ}$ 51, p. 43-65.

MÉNDEZ, R.; MICHELINI, J. J.; PRADA, J. y TÉBAR, J. Economía creativa y desarrollo urbano en España: una aproximación a sus lógicas espaciales. EURE. Revista Latinoamericana de Estudios Urbanos y Regionales, 2012, vol. 38, n 113, p. 5-32.

MÉNDEZ, R.; MICHELINI, J. J. y ROMEIRO, P. Redes socio-institucionales e innovación para el desarrollo de las ciudades intermedias. Ciudad y Territorio Estudios Territoriales, 2006, vol. XXXVIII, nº 148, p. 337-395. 
MÉNDEZ, R.; SÁNCHEZ, S.; ABAD, L. y GARCÍA, I. Dinámicas industriales, innovación y sistema urbano en España: trayectorias de las ciudades intermedias. Boletín de la Asociación de Geógrafos Españoles, 2008, n 46, p. 227-260.

MIGUÉLEZ, E y MORENO, R. Skilled labour mobility, networks and knowledge creation in regions: a panel data approach. Annals of Regional Science, 2013, n 51, p. 191-212.

MORGAN, K. The exaggerated death of geography: learning, proximity and territorial innovation systems. Journal of Economic Geography, 2004,vol. 4, nº 1, p. 3-21.

MOYLE, K. The hegemony of Microsoft: an Australian story. Prometheus, 2003, vol. 21, nº 2, p. 213-230.

MUKHERJI, N. y SILBERMAN, J. Absorptive capacity, knowledge flows, and innovation in U.S. metropolitan areas. Journal of Regional Science, 2013, vol. 53, nº 3, p. 392-417.

MUSCAR, E. F. La privatización como innovación: privación y exclusión social en Argentina. Scripta Nova, Revista Electrónica de Geografía y Ciencias Sociales. [En línea]. Barcelona: Universidad de Barcelona, 1 de agosto de 2000, no 69 (79). [4 de abril de 2013]. ISBN: 84-475$2488-4$.

OERLEMANS, L. A. G. y MEEUS, M. T. H. Do organizational and spatial proximity impact on firm performance? Regional Studies, 2005, vol. 39, n 1, p.89-104.

PALLARÉS, M. y VERA, A. Incrustación industrial y medio innovador en la comarca del Berguedà. En Alonso, J. L. y Méndez, R. (coordinadores). Innovación, pequeña empresa y desarrollo local en España. Madrid: Civitas ediciones, 2000.

PASCUAL, H. y GARCÍA, J. L. Políticas urbanas para el desarrollo y la innovación en las ciudades intermedias. Investigaciones Geográficas, 2008, nº 47, p. 5-25.

PIKE, A.; RODRÍGUEZ-POSE, A. y TOMANEY, J. Desarrollo local y regional. Valencia: PUV, 2011.

RODRÍGUEZ-POSE, A. y COMPTOUR, F. Do clusters generate greater innovation and growth? An analysis of European regions. The professional Geographer, 2012, vol. 64, no 2, p. 211-231.

SALOM, J. Política industrial de apoyo a la innovación en áreas de desarrollo endógeno: el caso de la Comunidad Valenciana a la luz de las experiencias europeas recientes. Cuadernos de Geografía, 1997, nº 61, p. 145-157.

SALOM, J. Innovación y actores locales en los nuevos espacios económicos: un estado de la cuestión. Boletín de la Asociación de Geógrafos Españoles, 2003, nº 36, p. 7-30. 
SÁNCHEZ, H. Microsoft Ibérica en la economía española. [En línea]. $<$ http://blogs.technet.com/b/hectormontenegro/archive/2009/10/06/microsoft-ib-rica-en-laeconom-a-espa-ola.aspx>, 2009. [24 de mayo de 2013].

SÁNCHEZ, J. E. Espacio, economía y sociedad. Madrid: Siglo XXI, 1991.

SANDLER, L. A. y BLANK, P. The quest to make accessibility a corporate article of faith at Microsoft: case study of corporate culture and human resource dimensions. Behavioral Sciences and the Law, 2005, vol. 23, p. 39-64.

SIMMIE, J. Innovation and urban regions as national and international nodes for the transfer and sharing of Knowledge. Regional Studies, 2003, nº. 37, p. 607-620.

SLATER, R. Microsoft rebooted: how Bill Gates and Steve Ballmer reinvented their company. New York: Penguin Group, 2004.

STROSS, R. The Microsoft way: the real story of how the company outsmarts its Competition. Nueva York: Basic Books, 1997.

STROSS, R. Planet Google. One company's audacious plan to organize everything we know, Nueva York: Free Press, 2009.

SUBRAMANIAN, S. EU obligation to the TRIPS agreement: EU Microsoft decision. European Journal of International Law, 2011, vol. 21, nº 4, p. 997-1.023.

TÖDTLING, F y KAUFMANN, A. SMEs in regional innovation systems and the role of innovation support. The case of Upper Austria. Journal of Technology Transfer, 2002, vol. 27, $\mathrm{n}^{\circ}$ 1, p. 15-26.

TORRE, A. y RALLET, A. Proximity and localization. Regional Studies, 2005, vol. 39, nº 1, p. 47-59.

TOULOUSE, S. A Microsoft Life. Joanner Starer, 2010.

TRANOS, E. y NIJKAMP, P. The death of distance revisited: cyber-place, physical and relational proximities. Journal of Regional Science, 2013, vol. 53, n 2, p. 864-873.

TROMPENAARDS, F y HAMPDEN-TURNER, C. Innovación en tiempos de crisis. Madrid: LID, 2010.

VILADECANS-MARSAL, E. y ARAUZO-CAROD. Can a knowledge-based cluster be created? The case of the Barcelona 22@ district. Papers in Regional Science, vol. 91, n², p. $377-401$.

VESPAGEN, B. y SCHOENMAKERS, W. The spatial dimension of patenting by multinationals firms in Europe. Journal of Economic Geography, 2004, vol. 4, nº 1, p. 23-42. 
WALLACE, J. y ERICKSON, J. Hard drive. Bill Gates and the making of the Microsoft empire. Nueva York: HarperCollins, 1993.

XIFRA, J. La gestión de conflictos potenciales como estrategia de comunicación corporativa: el caso de Microsoft. Zer Journal of Comunication Studies, 2005, nº 18, p. 67-81.

(C) Copyright Fernando Moliní, 2015.

(C) Copyright: Eduardo Estrada, 2015

(C) Copyright Scripta Nova, 2015.

Ficha bibliográfica:

MOLINÍ, F. ESTRADA, E. La innovación y la importancia de la cercanía o la lejanía: el caso de Microsoft Consulting Services. Scripta Nova. Revista Electrónica de Geografía y Ciencias Sociales. [En línea]. Barcelona: Universidad de Barcelona, 1 de diciembre de 2015, vol. XIX, no 524. <http://www.ub.es/geocrit/sn/sn-524.pdf>. ISSN: $1138-9788$. 